\title{
Phantom Bethe excitations and spin helix eigenstates in integrable periodic and open spin chains
}

\author{
Vladislav Popkov, ${ }^{1,2}$ Xin Zhang, ${ }^{1}$ and Andreas Klümper ${ }^{1}$ \\ ${ }^{1}$ Department of Physics, University of Wuppertal, Gaussstraße 20, 42119 Wuppertal, Germany \\ ${ }^{2}$ Faculty of Mathematics and Physics, University of Ljubljana, Jadranska 19, SI-1000 Ljubljana, Slovenia
}

\begin{abstract}
We demonstrate the existence of a special chiral "phantom" mode with some analogy to a Goldstone mode in the anisotropic quantum $X X Z$ Heisenberg spin chain. The phantom excitations contribute zero energy to the eigenstate, but a finite fixed quantum of momentum $k_{0}$. The mode exists not due to symmetry principles, but results from non-trivial scattering properties of magnons with momentum $k_{0}$ given by the anisotropy via $\cos k_{0}=\Delta$. Different occupations of the phantom mode lead to energetical degeneracies between different magnetization sectors in the periodic case. This mode originates from special string-type solutions of the Bethe ansatz equations with unbounded rapidities, the phantom Bethe roots (PBR). We derive criteria under which the spectrum contains eigenstates with PBR, both in open and periodically closed integrable systems, for spin $1 / 2$ and higher spins, and discuss the respective chiral eigenstates. The simplest of such eigenstates, the spin helix state which is a periodically modulated state of chiral nature, is built up from the phantom excitations exclusively. Implications of our results for experiments are discussed.
\end{abstract}

Interacting quantum spin systems are a vibrant research field as fascinating kinds of order are realized with rather complex order parameters or of topological nature. Even the spin$1 / 2 X X Z$ chain, despite its long history and being one of the best studied paradigmatic models in quantum statistical mechanics [1], remains a source of inspiration and fascinating new progress. This model is integrable and in principle allows for the calculation of objects that in generic systems are usually not accessible in the thermodynamic limit. Among the relatively recent results the discovery of a set of quasi-local conserved quantities [2] with strong implications on the theory of finite-temperature quantum transport [3] and successes in the calculation of finite temperature correlation functions $[4,5]$ are exciting achievements.

In this letter we are interested in phenomena of anisotropic quantum spin chains requiring the understanding of energetical degeneracies in uncharted territory. A first example is the physics of so-called spin helix states (SHS) (4) which show sharp local polarization with respect to site dependent axes. These states are routinely created, and widely used in coherent experimental protocols [6-8]. SHS can also be generated as non-equilibrium steady states via a dissipative quantum protocol [9-11] or via controlled local boundary dissipation. Remarkably, the needed boundary dissipation is of the type which allows the system to retain, partly, its integrability [12].

The eigenvalue degeneracies of isotropic quantum spin chains are well understood on the basis of the $s u(2)$ symmetry algebra. Simple eigenstates, that are fully polarized with respect to any axis form a multiplet of degeneracy $N+1$ for the spin-1/2 chain of length $N$.

The high degeneracy of this ferromagnetic multiplet can alternatively be explained by magnon excitations with soft Goldstone mode at wavenumber $k=0$. Contrary to the usual situation when all magnons carry different momenta, this precise $k=0$ mode can be occupied up to $N$ times.

A $z$-anisotropy of the spin exchange interaction lifts the high degeneracy, leaving just two degenerate eigenstates with spins fully polarized in $+z$ or $-z$ direction. The $s u(2)$-type degeneracies can be restored by the so-called "quantum defor- mation" $U_{q}(s u(2))$ of the symmetry algebra [13-15], involving special possibly non-hermitian boundary terms.

Remarkably, an analogue of a Goldstone mode scenario can happen in periodic spin systems with $z$-exchange anisotropy, namely a multiple occupation of a single mode can occur, but now with a nonzero wave vector $k_{0}$ fine-tuned to the system's anisotropy $J_{z} / J_{x}=\Delta$ via $\cos k_{0}=\Delta$. The corresponding excitation can be created at zero energetic cost. Like in the isotropic case, the possibility of multiple occupations of the same zero-energy phantom mode leads to the high degeneracy. Unlike in the isotropic case, the eigenstates form a multiplet of degenerate chiral states carrying finite current.

Excitations with momentum mode $\pm k_{0}$ were discussed in [16-21] for accounting for the energetical degeneracies of the spin-1/2 XXZ chain and related systems. For certain systems with commensurable values of $k_{0}$ extended symmetry algebras are realized and the completeness of the Bethe ansatz has been investigated [17-21].

In our letter we show why a macroscopic occupation of precisely $\pm k_{0}$ becomes possible, despite magnons of the wave number $k_{0}$ have non-trivial scattering. These states are realized by non-standard string-type solutions of the Bethe ansatz equations with infinite rapidities. The Bethe Ansatz equations for singular roots are satisfied with a universal choice for their arrangement (11), which makes them effectively "disappear" from the set of Bethe Ansatz equations. For this reason we call the roots with infinite rapidities phantom Bethe roots and the respective excitations phantom excitations.

We find phantom Bethe roots in other integrable systems including open quantum systems and also for higher spins.

Finally, we find that the role of the fully polarized eigenstates in the isotropic case is taken, in anisotropic systems, by simple but rather nontrivial chiral states, the spin helix states. The SHS have ballistic current and a harmonic modulation (with period $2 \pi / k_{0}$ ) of transversal magnetization. Remarkably, the SHS are created with exclusively phantom excitations, both in open and in periodic spin chains.

Factorized eigenstates at commensurate values of anisotropy. We consider the $X X Z$ spin-1/2 Hamiltonian for 
periodic and open boundary conditions. For the periodically closed chain we have

$$
\begin{aligned}
& H_{X X Z}=\sum_{n=1}^{N} h_{n, n+1}(\Delta), \\
& h_{n, n+1}(\Delta)=J\left[\sigma_{n}^{x} \sigma_{n+1}^{x}+\sigma_{n}^{y} \sigma_{n+1}^{y}+\Delta\left(\sigma_{n}^{z} \sigma_{n+1}^{z}-I\right)\right],
\end{aligned}
$$

with boundary conditions $\vec{\sigma}_{N+1} \equiv \vec{\sigma}_{1}$. For convenience we put $J=1$ throughout this letter. For the open chain we have

$$
H_{X X Z}=\sum_{n=1}^{N-1} h_{n, n+1}(\Delta)+\vec{h}_{l} \vec{\sigma}_{1}+\vec{h}_{r} \vec{\sigma}_{N}
$$

with boundary fields $\vec{h}_{l}$ and $\vec{h}_{r}$ on the first and on the last sites. In both cases a shift $-J \Delta$ in the nearest-neighbour interaction (1) is added for convenience. Both models (1), (2) are integrable and solvable via Bethe Ansatz methods [22-24]. We parametrize the anisotropy $\Delta$ of the exchange interaction as $\Delta=\cos \gamma$ or $\Delta=\cosh \eta$ with $\eta=\mathrm{i} \gamma$.

We want to construct factorized eigenstates of the Hamiltonians and introduce for each site the qubit state

$$
|y\rangle=\left(\begin{array}{c}
1 \\
\mathrm{e}^{y}
\end{array}\right)
$$

The qubit state (3) with $y=f+\mathrm{i} F$ corresponds to a fully polarized spin $1 / 2$ pointing into the direction $\vec{n}=$ $(\sin \theta \cos F, \sin \theta \sin F, \cos \theta)$ with $\tan \frac{\theta}{2}=\mathrm{e}^{f}$. A sitefactorized state, the so-called spin-helix state (SHS) $[9,10]$

$$
\left|S H S\left(y_{0}, \varphi\right)\right\rangle=\left|y_{0}\right\rangle_{1}\left|y_{0}+\mathrm{i} \varphi\right\rangle_{2} \ldots\left|y_{0}+\mathrm{i}(N-1) \varphi\right\rangle_{N},
$$

subscripts indicating the site number, with uniformly increasing angles on some offset $y_{0}$ becomes an eigenstate of the $X X Z$ Hamiltonian if: (i) the increase $\varphi$ of the angle is identical to $\pm \gamma$, the parameter of the anisotropy $\Delta=\cos \gamma$, and (ii) the boundary conditions can be accounted for. The parameter $\varphi$ is real (imaginary) for easy plane (easy axis) anisotropy corresponding to a state with uniformly increasing azimuthal (polar) angle.

The bulk interaction of the $X X Z$ Hamiltonian applied to any SHS state (4) results in 0 due to the "divergence" relation

$$
\begin{aligned}
& h(\Delta)|y\rangle \otimes|y+\mathrm{i} \gamma\rangle= \\
& \quad=|y\rangle \otimes\left(\kappa \sigma^{z}|y+\mathrm{i} \gamma\rangle\right)-\left(\kappa \sigma^{z}|y\rangle\right) \otimes|y+\mathrm{i} \gamma\rangle,
\end{aligned}
$$

where $\kappa=i \sin \gamma$. For the periodic model (1), the SHS will be an eigenstate if the periodic closure condition $\gamma N=$ $2 \pi m$ with integer $m$ is satisfied. This can only happen for anisotropy $|\Delta| \leq 1$.

For the open chain condition (ii) on the boundary can be satisfied not only in the case $|\Delta| \leq 1$, but also for $|\Delta|>1$. For $|\Delta|>1$ we may use expression (4) with the replacement $\varphi=\mathrm{i} \eta$ which results in a spin-helix state with fixed azimuthal angle and uniformly increasing polar angles. The eigenstate condition is fulfilled, if the boundary interactions $h_{l}=\vec{h}_{l} \vec{\sigma}_{1}$ and $h_{r}=\vec{h}_{r} \vec{\sigma}_{N}$ satisfy

$$
\begin{aligned}
h_{l}\left|y_{0}\right\rangle & =\kappa \sigma^{z}\left|y_{0}\right\rangle+\lambda_{-}\left|y_{0}\right\rangle, \\
h_{r}\left|y_{N-1}\right\rangle & =-\kappa \sigma^{z}\left|y_{N-1}\right\rangle+\lambda_{+}\left|y_{N-1}\right\rangle,
\end{aligned}
$$

where $y_{N-1}=y_{0}+\mathrm{i}(N-1) \gamma$, and $\lambda_{ \pm}$are some boundarydependent constants. The energy eigenvalue is $E=\lambda_{-}+\lambda_{+}$. Note that in the open chain case a condition on the anisotropy $\Delta$ like in the periodic case is absent and $\varphi$ in (4) can be real or imaginary. Although having the same algebraic form, the SHS for the easy plane and easy axis cases have rather different physical properties as visualized in Fig. 1.

The factorized SHS state is after the ferromagnetic state the simplest eigenstate of $X X Z$ spin chains. Yet the SHS (4) is quite nontrivial, and describes a "frozen" spin precession around the $z$-axis with period $2 \pi / \varphi$, see Fig. 1 . Due to the chiral nature, the SHS carries a remarkably high magnetization current, finite in the thermodynamic limit:

$$
\left\langle j^{z}\right\rangle_{S H S}=\left\langle 4 \mathrm{i}\left(\sigma_{n}^{+} \sigma_{n+1}^{-}-\text {h.c. }\right)\right\rangle_{S H S}= \pm 2 \frac{\sin \gamma}{\cosh ^{2}\left(\operatorname{Re}\left[y_{0}\right]\right)},
$$

where the sign \pm corresponds to the choice $\varphi= \pm \gamma$ in (4). Remarkably, the SHS (4) with adjustable wavelength can be realized in cold atom experiments [6,7].

The very existence of an eigenstate (4) for the periodic spin chain, characterized by periodic modulations in the magnetization profile seems to contradict the $U(1)$ symmetry: $X X Z$ eigenvectors split in blocks with well defined values of the global magnetization $S^{z}=\sum_{n} \sigma_{n}^{z}$ and expectation values $\left\langle\sigma_{n}^{+}\right\rangle=\left\langle\sigma_{n}^{-}\right\rangle=0$ vanish, and so do $\left\langle\sigma_{n}^{x}\right\rangle=\left\langle\sigma_{n}^{y}\right\rangle=0$.

This paradox is resolved by the energetical degeneracy of eigenstates with different values of the total magnetization $S^{z}$. We will show that a superposition of states from different blocks yields the state (4) which is not an eigenstate of the operator $S^{z}$.

Phantom Bethe roots at commensurate anisotropies in periodic $X X Z$ chains. The eigenstates and eigenvalues are given in terms of rapidities $\mu_{j}(j=1,2, \ldots n)$ whose total number $n$ may take any value out of $0,1, \ldots N$. For any solution of the Bethe Ansatz equations (BAE)

$$
\frac{\sinh ^{N}\left(\mu_{j}-\mathrm{i} \gamma / 2\right)}{\sinh ^{N}\left(\mu_{j}+\mathrm{i} \gamma / 2\right)}=\prod_{l \neq j}^{n} \frac{\sinh \left(\mu_{j}-\mu_{l}-\mathrm{i} \gamma\right)}{\sinh \left(\mu_{j}-\mu_{l}+\mathrm{i} \gamma\right)}
$$

there is an eigenstate with energy and total momentum

$$
E=-\sum_{j=1}^{n} e\left(\mu_{j}\right), \quad K=\sum_{j=1}^{n} k\left(\mu_{j}\right)
$$

with single particle energy and momentum defined by

$$
e\left(\mu_{j}\right)=\frac{4 \sin ^{2} \gamma}{\cosh \left(2 \mu_{j}\right)-\cos \gamma}, \quad \mathrm{e}^{\mathrm{i} k(\mu)}=\frac{\sinh \left(\mu+\mathrm{i} \frac{\gamma}{2}\right)}{\sinh \left(\mu-\mathrm{i} \frac{\gamma}{2}\right)} .
$$


The Bethe eigenvector $\Psi_{\mu_{1}, \ldots \mu_{n}}=B\left(\mu_{1}\right) \ldots B\left(\mu_{n}\right)|0\rangle$ is obtained by the application of magnon creation operators $B\left(\mu_{j}\right)$ to the reference state $|0\rangle=|\uparrow \uparrow \ldots \uparrow\rangle$ of fully polarized spins $[23,25]$.

Definition. We shall call a Bethe root $\mu_{p}$ satisfying (8), a phantom Bethe root, if it does not give a contribution to the respective energy eigenvalue (9) i.e. if $\operatorname{Re}\left[\mu_{p}\right]= \pm \infty$. The next Lemma affirms that such phantom Bethe roots do exist: Lemma 1: For anisotropy $\gamma=2 \pi m / N$ with integer $m$ there exist the following "phantom" solutions of the BAE (8) for any given $n=1,2, \ldots N$

$$
\mu_{p}= \pm \infty+\mathrm{i} \pi \frac{p}{n}, \quad p=1,2 \ldots n .
$$

These distributions remind of the string solutions to the Bethe ansatz equations. Note however that (11) holds for any finite system size $N$ with a total number $n$ of roots equidistantly distributed with separation $\pi / n$. Note that our lemma describes the precise arrangement of the infinite roots appearing in [1-6]. Upon introducing a finite magnetic flux resp. twisted boundary conditions, the roots become finite while the imaginary parts stay close to the values of Lemma 1 . This is relevant for the dependence of the energy as function of the twist and has important consequences for the transport properties, see [25].

Proof. Assume $\mu_{j}= \pm \mu_{\infty}+\mathrm{i} \pi j / n$, where $\mu_{\infty}$ has a large real part which we let to $\infty$ when evaluating the LHS of the Bethe ansatz equations. As $\gamma=2 \pi m / N$ the LHS of (8) becomes LHS $\rightarrow \mathrm{e}^{\mp \mathrm{i} \gamma N}=1$. On the RHS the term $\mu_{\infty}$ drops out leaving finite differences $\mu_{j}-\mu_{l}=\mathrm{i} \pi(j-l) / n$. Denoting $\omega=\mathrm{e}^{\mathrm{i} \pi / n}$, we have

$$
\begin{aligned}
& \mathrm{RHS}_{j}=\prod_{\substack{l=1 \\
l \neq j}}^{n} \frac{\omega^{j-l} \mathrm{e}^{-\mathrm{i} \gamma}-\omega^{-(j-l)} \mathrm{e}^{\mathrm{i} \gamma}}{\omega^{j-l} \mathrm{e}^{\mathrm{i} \gamma}-\omega^{-(j-l)} \mathrm{e}^{-\mathrm{i} \gamma}} \\
& =\prod_{l=1}^{n-1} \frac{\omega^{l} \mathrm{e}^{-\mathrm{i} \gamma}-\omega^{-l} \mathrm{e}^{\mathrm{i} \gamma}}{\omega^{l} \mathrm{e}^{\mathrm{i} \gamma}-\omega^{-l} \mathrm{e}^{-\mathrm{i} \gamma}}=\prod_{l=1}^{n-1} \frac{\omega^{l} \mathrm{e}^{-\mathrm{i} \gamma}-\omega^{-l} \mathrm{e}^{\mathrm{i} \gamma}}{-\omega^{-l} \mathrm{e}^{\mathrm{i} \gamma}+\omega^{l} \mathrm{e}^{-\mathrm{i} \gamma}}=1 .
\end{aligned}
$$

Here we used that the set of $\omega^{j-l}$ with $l=1, \ldots, n$ (and $\neq j$ ) is identical to the set of $\omega^{l}$ with $l=1, \ldots, n-1$ as we have $\omega^{n}=-1$.

Phantom Bethe vectors for periodic chains. The Bethe vectors corresponding to the phantom Bethe roots (PBR) solution (11), under the conditions of Lemma 1, can be constructed as described below (10). The two signs \pm in (11) correspond to different Bethe vectors which upon normalization read

$$
\begin{aligned}
& | \pm, n\rangle=\frac{1}{n ! \sqrt{\left(\begin{array}{c}
N \\
n
\end{array}\right)}} \sum_{l_{1}, \ldots, l_{n}=0}^{N-1} \mathrm{e}^{ \pm \mathrm{i} \gamma\left(l_{1}+\ldots+l_{n}\right)} \sigma_{l_{1}}^{-} \ldots \sigma_{l_{n}}^{-}|0\rangle \\
& n=0,1, \ldots, N
\end{aligned}
$$

Each multiplication by a $B\left(\mu_{j}\right)$-operator adds a quasiparticle with momentum $k\left(\mu_{j}\right)$ and zero energy. Within the standard picture [23, 24] quasi-particles obey a "Fermi rule": all $k\left(\mu_{j}\right)$ are usually different. This property is violated for phantom
Bethe roots $\mu_{p}$ for which all $k\left(\mu_{p}\right)$ are exactly the same: either $k\left(\mu_{p}\right)=+\gamma \equiv k_{0}$ or $k\left(\mu_{p}\right)=-\gamma \equiv-k_{0}$ depending on the sign of the singular part in (11). Repeated action of $B$ generates "phantom" Bethe states (12) with "quantized" momenta $\pm n \gamma$ and zero energy for all magnetization sectors $n$, yielding the degeneracy of the eigenvalue $E=0$ between different sectors. Note that the $E=0$ state is not a groundstate of (1), which is obtained by filling the Fermi sea with quasiparticles giving negative energy contributions to (9). The dimension of the degenerate subspace is $\operatorname{deg}=2(N-1)+2=2 N$ since the states $|+, n\rangle,|-, n\rangle$ for $n=1,2, \ldots N-1$ are linearly independent and for $n=0, N$ the states $|+, n\rangle,|-, n\rangle$ coincide. The degeneracy between sectors with different magnetization leads to eigenstates with periodic modulations in the density profile. Indeed, the SHS (4) with positive chirality and $\varphi=+\gamma=2 \pi m / N \neq \pi$ is a linear combination of phantom Bethe states $|+, n\rangle$, and SHS (4) with opposite chirality $\varphi=-\gamma$ is a linear combination of $|-, n\rangle$

$$
\left|S H S\left(y_{0}, \pm 2 \pi m / N\right)\right\rangle=\left(\begin{array}{c}
N \\
n
\end{array}\right)^{1 / 2} \sum_{n=0}^{N} \mathrm{e}^{y_{0} n}| \pm, n\rangle,
$$

see Supplemental [25] for the proof. Finally, note that the states (12) are chiral, which is evidenced by nonzero expectation values of the magnetization current, see Supplemental material [25].

$$
\left\langle \pm, n\left|j^{z}\right| \pm, n\right\rangle= \pm \frac{8 n(N-n)}{N(N-1)} \sin \gamma,
$$

reaching its maximum of order $\left|j^{z}\right| \rightarrow 2 \sin \gamma$ for $n=N / 2$.

Mixtures of regular and phantom excitations for the periodic XXZ model. Here we show that phantom Bethe roots can appear alongside with usual finite Bethe roots, for other special values of the anisotropy.

Let us assume that within a sector of $n_{0}$ flipped spins, there exists a BAE solution with $n$ phantom Bethe roots $\mu_{1}, \ldots, \mu_{n}$ and the remaining $r=n_{0}-n$ Bethe roots are regular. We denote the regular roots as $x_{1}, \ldots, x_{r}$ where $x_{j}=\mu_{n+j}$. Let us consider separately the BAE (8) subsets for phantom $\mu_{p}$ and for regular $x_{j}$. Substituting (11) in (8) we obtain

$$
\mathrm{e}^{\mathrm{i} \gamma(N-2 r)}=1,
$$

since each factor of the RHS containing a mixed pair $\mu_{p}, x_{j}$ contributes a term $\exp (2 \mathrm{i} \gamma)$. The product over factors of the RHS involving two phantom roots results in +1 precisely as in Lemma 1. The criterion (15) fixes the anisotropy parameter while the BAE subset for regular roots simplifies to

$$
\frac{\sinh ^{N}\left(x_{j}-\mathrm{i} \gamma / 2\right)}{\sinh ^{N}\left(x_{j}+\mathrm{i} \gamma / 2\right)}=\mathrm{e}^{ \pm 2 \mathrm{i} \gamma n} \prod_{\substack{l=1 \\ l \neq j}}^{r} \frac{\sinh \left(x_{j}-x_{l}-\mathrm{i} \gamma\right)}{\sinh \left(x_{j}-x_{l}+\mathrm{i} \gamma\right)},
$$

for all $j=1, \ldots, r$, see also $[19,20]$. This has the structure of the BAE of a twisted $X X Z$ chain, because of the presence of a constant phase factor. The signs \pm match those in (11). 
Phantom excitations in the open $X X Z$ chain. The energy of Hamiltonian (2) is given by (9) with an additional offset, $E=\sum_{j=1}^{N} e\left(\mu_{j}\right)+E_{0}$, where

$E_{0}=-\sinh \eta\left(\operatorname{coth} \alpha_{-}+\operatorname{coth} \alpha_{+}+\tanh \beta_{-}+\tanh \beta_{+}\right)$,

where the boundary fields $h_{l, r}$ are parametrized as

$\vec{h}=\frac{\sinh \eta}{\sinh \alpha_{ \pm} \cosh \beta_{ \pm}}\left(\cosh \theta_{ \pm}, \mathrm{i} \sinh \theta_{ \pm}, \mp \cosh \alpha_{ \pm} \sinh \beta_{ \pm}\right)$,

and $+(-)$ corresponds to the right (left) field. The Bethe roots $\mu_{j}$ satisfy BAE of a somewhat bulky form [25-28]. After some algebra [28] we find that if

$$
\begin{aligned}
\pm\left(\theta_{+}-\theta_{-}\right)= & (2 M-N+1) \eta+\alpha_{-}+\beta_{-}+\alpha_{+}+\beta_{+} \\
& \bmod 2 \pi \mathrm{i},
\end{aligned}
$$

is satisfied with some integer $M=0,1, \ldots, N-1$, each set of $N$ Bethe roots contains $n$ phantom Bethe roots of type (11), where $n$ takes one of two values $n_{+}=N-M$ and $n_{-}=M+1[28,29]$. The remaining $N-n$ Bethe roots $x_{j}$ $\left(=\mu_{n+j}\right)$ are regular and satisfy reduced BAE

$$
\begin{aligned}
& \frac{G_{ \pm}\left(x_{j}-\frac{\eta}{2}\right) \sinh ^{2 N}\left(x_{j}+\frac{\eta}{2}\right)}{G_{ \pm}\left(-x_{j}-\frac{\eta}{2}\right) \sinh ^{2 N}\left(x_{j}-\frac{\eta}{2}\right)}=\prod_{\substack{l=1 \\
l \neq j}}^{N-n_{ \pm}} \frac{\sinh \left(x_{j}-x_{l}+\eta\right)}{\sinh \left(x_{j}-x_{l}-\eta\right)} \times \\
& \times \frac{\sinh \left(x_{j}+x_{l}+\eta\right)}{\sinh \left(x_{j}+x_{l}-\eta\right)}, \quad j=1, \ldots, N-n_{ \pm}, \\
& G_{ \pm}(u)=\prod_{\sigma= \pm} \sinh \left(u \mp \alpha_{\sigma}\right) \cosh \left(u \mp \beta_{\sigma}\right),
\end{aligned}
$$

while the total eigenvalue has contributions from the regular Bethe roots only. We like to note that (16) holds literally for case $n=n_{+}$. For $n=n_{-}$the $+E_{0}$ contribution in (16) is to be replaced by $-E_{0}$, see [28]. We find that the BAE (18) for $n=N-M$ describes $\operatorname{dim} G_{M}^{+}=\sum_{m=0}^{M}\left(\begin{array}{l}N \\ m\end{array}\right)$ Bethe states, while the remaining $2^{N}-\operatorname{dim} G_{M}^{+}$eigenstates are contained in the other, complementary BAE set for $n=M+1$ [28, 29]. Unlike in the periodic setup, where some Bethe eigenstates contain PBR modes, and other eigenstates are fully regular, in open systems, satisfying criterion (17), all $2^{N}$ eigenstates include phantom Bethe roots. Remarkably, the condition (17) appears in [30-33] as a condition for the application of the Algebraic Bethe Ansatz. The BAE set (18) coincides with that found by an alternative method [30, 31, 33].

Now we focus on the simplest Bethe states, corresponding to all Bethe roots being phantom, $n_{+}=N$, the respective energy given by $E_{0}(16)$. We demonstrate that such "phantom" Bethe states are spin-helix states (4) with appropriately chosen parameters. The phantom Bethe states for mixtures of phantom and regular Bethe roots can be also obtained explicitly and show chiral features [28, 29].

Phantom Bethe states: open $X X Z$ chain. Easy plane regime $|\Delta|<1$. It is straightforward to verify that the
SHS (4), with $\varphi=\gamma, \operatorname{Re}\left[y_{0}\right]=\beta_{-}$and phase $\operatorname{Im}\left[y_{0}\right]=$ $\pi+\mathrm{i} \alpha_{-}+\mathrm{i} \theta_{-}$(note that $\alpha_{-}, \theta_{-}$are imaginary and $\beta_{-}=-\beta_{+}$ are real to ensure hermiticity of $H$ ), is an eigenstate of $H$. Indeed, one can check that (6), (7) are satisfied with $\lambda_{ \pm}=$ $-\sinh \eta\left(\operatorname{coth} \alpha_{ \pm}-\tanh \beta_{ \pm}\right)$, so that this SHS is an eigenvector of (2) with eigenvalue $\lambda_{-}+\lambda_{+}$, which coincides with the phantom Bethe vector eigenvalue $E_{0}$ (16). For the magnetization profile of this SHS see Fig. 1, top panel. Unlike for the periodic chain, here the eigenvalue $E_{0}$ is generically non-degenerate.

Simplest experimental setup. Using our results, longlived SHS can be obtained in experiments where effectively one-dimensional spin $1 / 2 X X Z$ chains with tunable anisotropy are realized [6, 7]. A spin helix of the form (4) with an adjustable wavelength is created within cold atoms setups by applying a magnetic field gradient in $z$ direction on an array of initially noninteracting qubits polarized along the $x$ axis, see Methods of [6] for details. To make the SHS an eigenstate of the $X X Z$ Hamiltonian, the wavelength $Q$ of the spin-helix and the $z$-anisotropy $\Delta$ must be related as $\Delta=\cos Q a$ where $a$ is the lattice constant. Indeed under this choice an SHS of type (4) $\left|S H S_{ \pm}\right\rangle:=\left|S H S\left(\mathrm{i} F_{0}, \pm Q a\right)\right\rangle$ will remain invariant in the bulk and change initially only at the boundaries, since

$$
\sum_{n=1}^{N-1} h_{n, n+1}(\Delta)\left|S H S_{ \pm}\right\rangle=\mp \mathrm{i} \sin Q a\left(\sigma_{1}^{z}-\sigma_{N}^{z}\right)\left|S H S_{ \pm}\right\rangle
$$

as follows from (5). The ends of the spin chain will thus play the role of defects, and the state in the bulk will be altered only by propagation of the information from the boundaries. Thus the state can be destroyed only after times of order $t=$ $\mathrm{Na} / v_{\text {char }}$, where $v_{\text {char }}$ is the sound velocity, $N$ is the number of spins and $a$ is the lattice constant. For example, in [6, 7], the process of the expansion of the defect in the bulk can be monitored. On the other hand, if the SHS period does not match the anisotropy $\Delta \neq \cos Q a$, then the initial SHS will be destroyed after times of order $t=a / v_{\text {char }}$. On one hand, the effect is robust (w.r.t. the phase of the helix and chain length $N$ ), and on the other hand, it is sensitive w.r.t. the matching condition for the anisotropy $\Delta$. This sensitivity can be used as a benchmark for calibrating the anisotropy, or the wave-length of the produced SHS, or both.

Phantom Bethe states: Easy axis $\Delta=\cosh \eta>1$. The SHS of the form (4) with $y_{0}=\mathrm{i} \pi-\theta_{-}+\alpha_{-}+\beta_{-}$satisfies (6), (7) with $\kappa \rightarrow-\sinh \eta$ and $\lambda_{ \pm}=-\sinh \eta\left(\operatorname{coth} \alpha_{ \pm}+\right.$ $\left.\tanh \beta_{ \pm}\right)$. Consequently, state (4) is an eigenstate of $H$ with eigenvalue $\lambda_{+}+\lambda_{-}=E_{0}$. Thus, state (4) is a phantom Bethe vector. It describes spins on the lattice with fixed azimuthal angle and changing polar angle along the chain, see Fig. 1, lower panel. Unlike the "azimuthal" spin helix state (4), the "polar" SHS carries no spin current, $\left\langle j^{z}\right\rangle_{S H S_{\text {polar }}}=0$.

Discussion. We have described a novel scenario of excitations in integrable systems, namely phantom excitations with phantom Bethe roots corresponding to unbounded rapidities. The existence criterion for these states is formulated and de- 


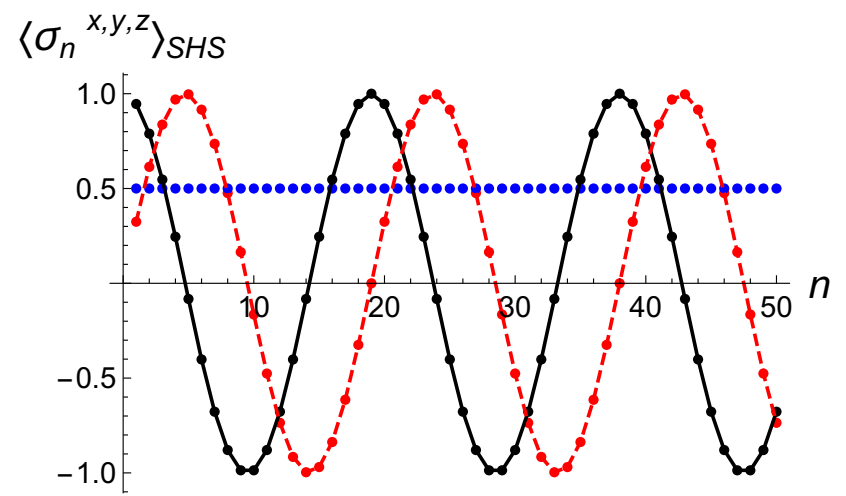

$\left\langle\sigma_{n}{ }^{x, y, z}\right\rangle_{S H S_{p o l a r}}$

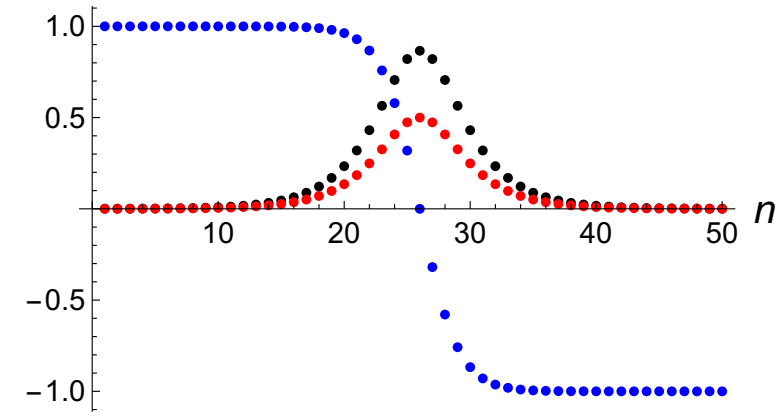

Figure 1. Components of local magnetization $\left\langle\sigma_{n}^{x}\right\rangle,\left\langle\sigma_{n}^{y}\right\rangle,\left\langle\sigma_{n}^{z}\right\rangle$ for SHS/phantom Bethe states versus site number $n$, for the easy plane (upper panel) and the easy axis case (lower panel), indicated with black, red and blue points respectively. Upper panel: SHS (4) with increasing azimuthal angle, the phantom Bethe eigenstate of (2) or (1) for $|\Delta|<1$. Parameters: $\varphi=\gamma=2 \pi / 19, y_{0}=\mathrm{i} \gamma+1 / \sqrt{3}$. Curves connecting points serve as a guide for the eye. Lower panel: SHS (4) with increasing polar angle, the phantom Bethe eigenstate of (2) for $\Delta>1$. Parameters: $\mathrm{i} \varphi=\eta=2 \pi / 19, y_{0}=\mathrm{i} \pi / 6+N \eta / 2$.

pends on the boundary conditions of the system. Under this criterion a certain subset of Bethe roots is located at infinity with relative positions at equidistant points. This resembles a perfect TBA string, but is of entirely different nature.

For models with periodic boundaries the PBR are responsible for degeneracies between sectors with different total magnetization, and lead to factorized spin helix eigenstates at anisotropies given by (15). Also for the open $X X Z$ model the PBR related eigenstates are spin helix states with winding polarization vector, in the easy plane regime, and the "polar angle"-version of the latter, in the easy axis regime. Our results can be used for the generation of stable spin helix states in experimental setups realizing $X X Z$ chains $[6,7]$.

While our discussion was restricted to the $X X Z$ model, the presence of phantom Bethe roots, due to their simple analytic form (11), can be easily established in other integrable models, e.g. in the periodic spin-1 Fateev-Zamolodchikov model [34-36], and arbitrary spin $s$ generalizations [37-40], see Supplemental Material [25]. It would be interesting to search for PBR analogues in intrinsically non-hermitian inte- grable models, e.g. [41, 42].

Financial support from the Deutsche Forschungsgemeinschaft through DFG project KL 645/20-1, is gratefully acknowledged. X. Z. thanks the Alexander von Humboldt Foundation for financial support. V. P. acknowledges support by European Research Council (ERC) through the advanced Grant No. 694544-OMNES. V. P. thanks S. Essink for indicating the work in [6]. We thank W. Ketterle for drawing our attention to his newest experiment [7], where the time evolution of a transversal spin helix state (4) has been studied.

[1] M. Gaudin. The Bethe Wavefunction (Cambridge University Press, Cambridge, 2014).

[2] T. Prosen, E. Ilievski. Families of quasilocal conservation laws and quantum spin transport. Phys. Rev. Lett. 111, 057203 (2013).

[3] B. Bertini, F. Heidrich-Meisner, C. Karrasch, T. Prosen, R. Steinigeweg, M. Znidaric. Finite-temperature transport in one-dimensional quantum lattice models. arXiv:2003.03334.

[4] F. Göhmann, A. Klümper, A. Seel. Integral representations for correlation functions of the XXZ chain at finite temperature. $J$. Phys. A: Math. Gen. 37, 7625 (2004).

[5] H. E. Boos, F. Göhmann, A. Klümper, J. Suzuki. Factorization of multiple integrals representing the density matrix of a finite segment of the Heisenberg spin chain. J. Stat. Mech. P04001 (2006).

[6] N. Jepsen, J. Amato-Grill, I. Dimitrova, W. W. Ho, E. Demler, W. Ketterle. Spin transport in a tunable Heisenberg model realized with ultracold atoms. Nature 588, 403 (2020).

[7] P. N. Jepsen, W. W. Ho, J. Amato-Grill, I. Dimitrova, E. Demler, W. Ketterle. Transverse spin dynamics in the anisotropic heisenberg model realized with ultracold atoms. arXiv:2103.07866.

[8] S. Hild, T. Fukuhara, P. Schauß, J. Zeiher, M. Knap, E. Demler, I. Bloch, C. Gross. Far-from-equilibrium spin transport in heisenberg quantum magnets. Phys. Rev. Lett. 113, 147205 (2014).

[9] V. Popkov, C. Presilla. Obtaining pure steady states in nonequilibrium quantum systems with strong dissipative couplings. Phys. Rev. A 93, 022111 (2016).

[10] V. Popkov, G. M. Schütz. Solution of the Lindblad equation for spin helix states. Phys. Rev. E 95, 042128 (2017).

[11] V. Popkov, T. Prosen, L. Zadnik. Exact Nonequilibrium Steady State of Open XXZ/XYZ Spin-1/2 Chain with Dirichlet Boundary Conditions. Phys. Rev. Lett. 124, 160403 (2020).

[12] T. Prosen. Matrix product solutions of boundary driven quantum chains. J. Phys. A: Math. Theor. 48, 373001 (2015).

[13] V. Drinfeld. Quantum groups. In A. M. Gleason(editor ) Proceedings of the International Congress of Mathematicians, vol. 1, 798 (Berkeley, CA, 1986).

[14] M. Jimbo. A $q$-difference analogue of $\mathrm{U}(\mathrm{g})$ and the YangBaxter equation. Lett. Math. Phys. 10, 63 (1985).

[15] M. Jimbo. A $q$-analogue of $\mathrm{U}(\mathrm{g}[(\mathrm{N}+1))$, Hecke algebra, and the Yang-Baxter equation. Lett. Math. Phys. 11, 247 (1986).

[16] V. Pasquier, H. Saleur. Common structures between finite systems and conformal field theories through quantum groups. Nucl. Phys. B 330, 523-556 (1990).

[17] T. Deguchi, K. Fabricius, B. McCoy. The sl(2) loop algebra symmetry of the six-vertex model at roots of unity. J. Stat. 
Phys. 102, 701-736 (2001).

[18] K. Fabricius, B. McCoy. Bethe's equation is incomplete for the XXZ model at roots of unity. J. Stat. Phys. 103, 647-678 (2001).

[19] D. Braak, N. Andrei. On the spectrum of the XXZ-chain at roots of unity. J. Stat. Phys. 105, 677-709 (2001).

[20] R. Baxter. Completeness of the Bethe ansatz for the six and eight-vertex models. J. Stat. Phys. .

[21] Y. Miao, J. Lamers, V. Pasquier. On the Q operator and the spectrum of the XXZ model at root of unity. arXiv:2012.10224.

[22] R. J. Baxter. Exactly solved models in statistical mechanics (Academic Press, London, 1982).

[23] L. A. Takhtadzhan, L. D. Faddeev. The quantum method of the inverse problem and the heisenberg XYZ model. Russ. Math. Surv. 34, 11 (1979).

[24] E. K. Sklyanin, L. A. Takhtadzhyan, L. D. Faddeev. Quantum inverse problem method. i. Theor. Math. Phys. 40, 688 (1979).

[25] See Supplemental material http://link.aps.org/supplemental/ 10.1103/PhysRevB.104.L081410 for the proof of Eqs. (13) and (14), explicit BAEs for open spin chain, and phantom Bethe roots in models with arbitrary spin. .

[26] Y. Wang, W.-L. Yang, J. Cao, K. Shi. Off-Diagonal Bethe Ansatz for Exactly Solvable Models (Springer, Berlin, 2015).

[27] X. Zhang, Y.-Y. Li, J. Cao, W.-L. Yang, K. Shi, Y. Wang. Bethe states of the XXZ spin-1/2 chain with arbitrary boundary fields. Nucl. Phys. B 893, 70 (2015).

[28] X. Zhang, A. Klümper, V. Popkov. Phantom Bethe roots in the integrable open spin- $\frac{1}{2}$ XXZ chain. Phys. Rev. B 103, 115435 (2021).

[29] X. Zhang, A. Klümper, V. Popkov, Chiral coordinate Bethe ansatz for phantom eigenstates in the open XXZ spin- $\frac{1}{2}$ chain . arXiv:2107.13266.

[30] J. Cao, H.-Q. Lin, K.-J. Shi, Y. Wang. Exact solution of XXZ spin chain with unparallel boundary fields. Nucl. Phys. B 663, 487 (2003).
[31] R. I. Nepomechie. Bethe ansatz solution of the open XXZ chain with nondiagonal boundary terms. J. Phys. A: Math. Gen. 37, 433 (2003).

[32] R. I. Nepomechie, F. Ravanini. Completeness of the Bethe Ansatz solution of the open XXZ chain with nondiagonal boundary terms. J. Phys. A: Math. Gen. 36, 11391 (2003).

[33] J. Cao, W.-L. Yang, K. Shi, Y. Wang. Off-diagonal Bethe ansatz solutions of the anisotropic spin-1/2 chains with arbitrary boundary fields. Nucl. Phys. B 877, 152 (2013).

[34] A. B. Zamolodchikov, V. A. Fateev. Model factorized S-matrix and an integrable spin-1 Heisenberg chain. Sov. J. Nucl. Phys. 32 (1980).

[35] P. P. Kulish, N. Y. Reshetikhin. Quantum linear problem for the sine-gordon equation and higher representations. J. Sov. Math. 23, 2435 (1983).

[36] L. Mezincescu, R. I. Nepomechie, V. Rittenberg. Bethe ansatz solution of the Fateev-Zamolodchikov quantum spin chain with boundary terms. Phys. Lett. A 147, 70 (1990).

[37] K. Sogo. Ground state and low-lying excitations in the Heisenberg XXZ chain of arbitrary spin S. Phys. Lett. A 104, 51 (1984).

[38] H. M. Babujian, A. Tsvelick. Heisenberg magnet with an arbitrary spin and anisotropic chiral field. Nucl. Phys. B 265, 24 (1986).

[39] A. N. Kirillov, N. Y. Reshetikhin. Exact solution of the integrable XXZ Heisenberg model with arbitrary spin. I. The ground state and the excitation spectrum. J. Phys. A: Math. Gen. 20, 1565 (1987).

[40] L. D. Faddeev. How algebraic Bethe ansatz works for integrable model (1996). arXiv:hep-th/9605187.

[41] A. A. Ziolkowska, F. H. Essler. Yang-Baxter integrable Lindblad equations. SciPost Phys. 8, 44 (2020).

[42] M. de Leeuw, C. Paletta, B. Pozsgay. Constructing Integrable Lindblad Superoperators. arXiv:2101.08279. 